\title{
A second traumatic spinal cord injury: associated risk factors. Case report and review
}

\author{
J A Sliwa DO, Ai Chi Lim MD, E J Roth MD
}

Department of Physical Medicine and Rehabilitation, Northwestern University Medical School, Rehabilitation Institute of Chicago, 345E Superior Street, Chicago, Illinois 60611, USA.

Despite advances in acute care and long term management, traumatic spinal cord injury remains a devastating and disabling event. This report describes the case of a second traumatic spinal cord injury in a previously rehabilitated and functionally independent paraplegic. Factors potentially associated with the second spinal cord injury in this patient include wheelchair use, previous spinal fusion, alcohol use and sensation-seeking behavior. These factors, which are common to many spinal cord injured patients, could potentially be risk factors for a second traumatic spinal injury.

Key words: spinal cord injuries; second spinal cord injury; wheelchairs; ethyl alcohol; spinal fusion; behavior.

\section{Introduction}

Damage to the spinal cord with resulting neurological sequelae can be a severely disabling event. It is estimated that over 6,000 spinal cord injuries (SCIs) occur annually and that just under 200,000 persons live with residual deficits from a spinal cord injury. ${ }^{1}$ Common causes of traumatic SCI include motor vehicle accidents, falls, diving, and gunshot wounds. Advances in acute care and long term management have improved both survival and life expectancy in these patients. ${ }^{2}$ Likewise, levels of functional independence have been found to improve with comprehensive rehabilitation. ${ }^{3,4}$ Despite these improvements, traumatic spinal cord injury remains a devastating event. Little, if anything, has been written about individuals who sustain a second traumatic spinal cord injury, and the successes and problems of comprehensive medical rehabilitation in such patients. This report describes a $\mathrm{T} 1$ paraplegic who had completed rehabilitation and was functioning independently in the community when he suffered a second traumatic spinal cord injury resulting in upper extremity motor loss. It also highlights several factors common to many spinal cord injured individuals that may be risk factors for a second injury to the spinal cord.

\section{Case report}

A 26 year old male, who was driving while intoxicated with alcohol, was involved in a motor vehicle accident resulting in a T2 vertebral compression fracture and $\mathrm{T} 1 \mathrm{com}$ plete sensory-motor paraplegia. $\mathrm{He}$ underwent posterior spinal fusion with Luque rod placement and iliac crest bone graft between C7 and T3. The postoperative period was complicated by a left pleural effusion which required placement of a chest tube. The patient was subsequently transferred to a rehabilitation facility for comprehensive interdisciplinary inpatient rehabilitation. Following an 11-week course of rehabilitation, he was discharged home able to feed, dress, and wash himself independently. He was also able to transfer, propel his wheelchair, catheterize his bladder, and perform his bowel program independently.

Two years following his initial injury, the patient sustained a second spinal cord injury. While returning home after an evening of drinking with friends, he was ascending a ramp to enter his home when he fell backwards in his wheelchair. He struck the back of his head and neck on the ramp, and immediately experienced neck pain, upper extremity paresthesiae and loss of grip strength bilaterally. The patient was transported to an emergency room where radio- 
graphic evaluation revealed a vertebral subluxation of $\mathrm{C} 5$ on $\mathrm{C} 6$ with a locked facet on the left. Gardner-Wells tongs with traction were applied, but when reduction could not be achieved the patient underwent an open reduction and a second posterior spinal fusion of $\mathrm{C} 5$ and $\mathrm{C} 6$ with iliac crest bone graft.

Following stabilization of the spine, the patient was transferred for a second course of comprehensive inpatient rehabilitation. General physical examination at the time of admission was unremarkable. However, manual muscle testing revealed new upper extremity muscle weakness as follows: the biceps had $2 / 5$ strength on the left and $5 / 5$ on the right; wrist extensors had $4 / 5$ strength on the left and $4 / 5$ on the right; triceps tested at $5 / 5$ strength bilaterally; finger flexors had $3 / 5$ strength bilaterally; and hand intrinsics displayed $2 / 5$ strength bilaterally. He had no voluntary movement of his trunk or lower extremity muscles and sensation remained intact only through the T1 dermatome. Functionally the patient required assistance for performance of all activities of daily living, transfers and wheelchair skills on admission.

The patient had a history of alcohol and drug usage, as well as of risk-taking behavior prior to his initial injury. Furthermore, during both inpatient rehabilitation admissions, the staff reported that the patient was frequently confrontational, demanding, non compliant and verbally abusive. The patient participated in therapy however, and made good progress with improvements in upper extremity strength and functional skills. At the time of discharge, he was independently performing oral-facial hygiene, bed mobility, wheelchair transfers, short distance wheelchair propulsion, and intermittent bladder catheterization. He required setup and equipment for feeding, and assistance for buttoning, lower extremity dressing and his bowel program.

\section{Discussion}

In 1978, Toerge and associates reported on secondary spinal fractures following a traumatic myelopathy. They reviewed 400 spinal cord injured patients and noted that 18 $(4.5 \%)$ had secondary noncontiguous spinal injuries that had occurred at the time of their initial injury. None of the secondary lesions produced a new or different motor level but were implicated in bladder, skin and postural complications. ${ }^{5}$ The patient reported here is atypical and unique in that he suffered a second, and separate, traumatic spinal cord injury which resulted in an increased degree of motor impairment and a decline in functional status. The second myelopathy at a more proximal level produced a decline in upper extremity functioning in a patient who had previously been a highly functioning paraplegic.

There are a number of factors that either individually or in combination could have contributed to the second injury in this patient. These risk factors need to be examined as being potential contributors to susequent injuries in other spinal cord injured patients. Although falls from a wheelchair are common and few patients sustain major injury, wheelchair use, both manual and motorized, is not without potential for serious injury. Paulson and associates reported a case of splenic rupture requiring splenectomy in a 22 year old quadriplegic who suffered abdominal trauma while being transferred to his wheelchair. ${ }^{6}$ Hays and associates reported the death from hypothermia, of an adolescent male with muscular dystrophy whose motorized wheelchair became inoperable due to a broken caster fork while the patient was returning home from visiting friends. ${ }^{7}$ In the patient reported here, there was no wheelchair malfunction or carelessness that could be attributed to a caregiver. Our patient had received comprehensive rehabilitation after his initial spinal cord injury which included training in advanced wheelchair skills. $\mathrm{He}$ had mastered these skills and had functioned safely and independently in the wheelchair. Despite this, he experienced a fall during routine use of his wheelchair that resulted in a second traumatic spinal cord injury.

A second factor that could conceivably have contributed to the severity of the second injury was the initial spinal fusion with Luque rod placement between $\mathrm{C} 7$ and 
T3 and the resulting altered spinal mechanics. Our patient fell backwards as he ascended a ramp in his wheelchair, striking the back of his head and causing excessive flexion of the cervical spine. With no movement at the previously fused segments, such forces could conceivably produce exaggerated movement at the proximal mobile cervical segments, resulting in disruption of the vertebral column and a traumatic myelopathy.

Thirdly, is the use of alcohol and its contribution to our patient's injuries. Frisbie and Tun surveyed 137 spinal cord injured patients and reported that $39(28 \%)$ of these drank an average of 6 drinks on the day of the injury. ${ }^{8}$ Questioning 30 consecutively admitted spinal cord injured patients, Fullerton and associates reported that 15 had been drinking at the time of their injuries and 5 admitted to being intoxicated immediately prior to their injury. ${ }^{9}$ Heinemann and associates studied alcohol use in 103 recently injured spinal cord patients and reported that $95 \%$ of those questioned acknowledged prior alcohol use. The average weekday quantity of alcohol consumed when drinking was 5.9 drinks. ${ }^{10}$ The patient reported here has a history of frequent alcohol usage. Furthermore, alcohol consumption preceded both injuries.

Lastly is the role of sensation-seeking behavior in traumatic spinal cord injury and specifically its contribution in our patient. Sensation-seeking individuals tend to be more extroverted, thrill-seeking, implusive, antisocial and noncomformist, with a tendency to injury, alcohol and drug abuse, and implusive behavior. ${ }^{11}$ Many of these characteristics were displayed by our patient. In a study comparing 140 SCI patients with 140 matched controls, Mawson and associates showed modest but statistically significant higher scores by the spinal cord injured in the disinhibition and boredom susceptibility parts of the Sensation Seeking Scale. The authors did conclude however, that 'sensation-seeking' as presently defined and measured was of limited potential usefulness in identifying people at risk for a spinal cord injury. ${ }^{11}$

The patient presented in this report returned to independent living following his first spinal cord injury, only to experience a second traumatic injury to the cervical cord 2 years later as a result of a fall from his wheelchair. The role of our patient's wheelchair usage, his previous spinal fusion, his alcohol usage and possible sensation-seeking behavior in the second injury can only be speculative. These 4 factors, common to many spinal cord injured patients, could have contributed to his second injury and thus could be considered risk factors for a second traumatic myelopathy. In this light, medical staff involved in the care of this patient population should be actively engaged in minimizing these risk factors. Little can be done for some of these factors such as the spinal fusion required by this patient for spinal stabilization, or intervention for sensation-seeking behavior. However, the prescription of safer wheelchairs with antitip devices, training patients to fall safely, and counselling of the frequent alcohol user are specific measures that can be utilized in an attempt to minimize the risks of a second traumatic spinal cord injury.

\section{References}

1 DeVivo MJ, Fine PR, Maetz HM, Stover SL (1980) Prevalence of spinal cord injury; a reestimation employing life table techniques. Arch Neurol 37: 707-708.

2 DeVivo MJ, Kartus PL, Stover SL, Rutt RD, Fine PR (1987) Seven-year survival following spinal cord injury. Arch Neurol 44: 872-875.

3 Yarkony GM, Roth EJ, Heinemann AW, Wu YC, Katz RT, Lovell L (1987) Benefits of rehabilitation for traumatic spinal cord injury; multivariate analysis in 711 patients. Arch Neurol 44: 93-96.

4 Woolsey RM (1985) Rehabilitation outcome following spinal cord injury. Arch Neurol 42: 116-119.

5 Toerge JE, Rosen JS, Calenoff L (1978) Secondary spinal lesions in spinal cord injury patients. Arch Phys Med Rehabil 59: 343-345.

6 Paulson SM, Hatvani C, Long C (1983) Splenic rupture and splenectomy due to fall from wheelchair. Arch Phys Med Rehabil 64: 180-181.

7 Hays RM, Jaffe KM, Ingman E (1985) Accidental death associated with wheelchair use: a case report. Arch Phys Med Rehabil 66: 709-710. 
8 Frisbie JH, Tun CG (1984) Drinking and spinal cord injury. J Am Para Soc 7: 71-73.

9 Fullerton DT, Harvey RF, Klein MH, Howell T (1981) Psychiatric disorders in patients with spinal cord injuries. Arch Gen Psychiatry 38: 1368-1371.

10 Heinemann AW, Donohue R, Keen M, Schnoll S (1989) Alcohol use by persons with recent spinal cord injuries. Arch Phys Med Rehabil 69: 619-624.

11 Mawson AR, Jacobs KW, Winchester Y, Biundo JJ, Jr (1988) Sensation-seeking and traumatic spinal cord injury: case-control study. Arch Phys Med Rehabil 69: 1039-1043. 